\title{
CONTROL OF AGRICULTURAL LAND POLLUTION DUE TO EXTENSIVE USE OF PESTICIDE AND INSECTICIDE BY BACTERIA CULTURE
}

\author{
Tanusri Mandal $^{1}$, Sangeeta Saha ${ }^{2}$, N. K. Mandal ${ }^{3}$
}

\begin{abstract}
Experimental studies have been carried out to find the application of bacteria culture to control the pollution of agricultural lands by extensive use of insecticide and pesticide by the farmers to protect their crops. For this, two bacterial strains, Enterobacter sp.,named Z57, and Pseudomonas sp., named Es20, having NCBI accession number KC169992 and KCL169988 has been used after isolated from cow dung. It can degrade calcium phytate, chlorpyrios, methayl-parathion and phorate. It has also been seen that molybdenum salt can act as the catalyst for enhancement of phorate degradation. Different types of tests, like- pH measurement, phosphate solubilizing efficiency test, Quantative estimation of soluble phosphate , Kinetics for Biodegradation of phorate, Plant growth promotion test, Bio-fertilizer test and analysis like- Biochemical characterization, and LC-MS analysis have been done to find the application of bacteria to biodegrade toxic water-insoluble organophosphorus compounds so that pollution of the agricultural lands can be controlled. Data of the Results have been analyzed and discussed.

Key words: Bacteria; Organophosphate; Biodegradation; Bio-fertilizer; Land pollution.
\end{abstract}

\section{INTRODUCTION}

Control of pollution of agricultural lands due to contamination of pesticides and insecticides has been given due importance for the last many years[1,2 ]. This due to the facts that: i) it is causing the agricultural lands in-fertile and useless for further corps production, ii) cost of crop production becoming high, iii) top- soil is required to remove and fill with fresh soil for to make it useful iv) due to current of water in rainy season, it pollutes other lands and vi) reduction in crop yield, etc..

Phosphorous is an essential macronutrient for the growth of the plant. It has important role in various processes. It has been reported that plantroot can absorb only $0.1 \%$ of the total phosphorus contained in the soil [3]. Hence, phosphorus deficiency has been supplemented with synthetic chemical fertilizers for green revolution. Along with synthetic phosphate fertilizer, other organophosphates, such as pesticides, insecticides and acaricides are used for protection of crops. Such organophosphate compounds are toxic in nature. These toxic compounds damage the soil quality, plant growth rate and microbial diversity[6]. According to report, crop-yield is reduced by $45 \%$ due to the excessive application of pesticides[4]. The isolated bacterium can be used to degrade five different types of organophosphate insecticide compounds. Three toxic organo-phosphorus insecticides which are also used in agricultural field can also be degraded by bacteria[5]. In this paper, an attempt has been made to report the results of experiments done to study the effect of bacteria culture to control the pollution of the agricultural field due to extensive use of pesticides and insecticides.

\section{MATERIALS AND METHODS}

\subsection{Isolation of Bacteria:}

Locally available cow dung has been used to isolate two bacterial strains, ZS20 and E57 . Their NCBI accession numbers are KCL169992 and KCL169988 respectively. They have been used for pollution control of agricultural lands.

\subsection{Chemical Reagent used:}

The main reagent, phorate having trade mark name- Tilmet 10G of Shubham Spray India Limited has been used for the experiment. Other chemical reagents with analytical grade of standard commercial companies have been used for this investigation.

\subsection{Broth assay:}

Broth cultures of two different bacterial strains have been prepared for quantitative assessment of $\mathrm{pH}$, bacterial growth, solubilizing efficiency, biodegradation of phorate and plant growth promotion. In addition, the catalytic effect of molybdenum salts on phorate degradation has been studied.

\footnotetext{
${ }^{1}$ OIST, Vidyasagar University, Midnapore, West Bengal, India

${ }^{2}$ OIST, Vidyasagar University, Midnapore, West Bengal, India

${ }^{3}$ University of Engineering \& Management, Kolkata, India.
} 


\section{$2.4 \mathrm{pH}$ measurement of different broth:}

In this experiment we have studied twelve cases, six cases for each bacterial strain. The $\mathrm{pH}$ of each bacterial strain and $\mathrm{pH}$ of its respective control supernatant have been separately measured with Digital $\mathrm{pH}$ meter. The readings have been measured on every other day starting from 2 nd to 16 th day with respect to the day of inoculation.

\subsection{Bacterial growth measurement:}

$2 \mathrm{ml}$ of each broth culture of each bacterial has been taken on every other day starting from 2nd to 18th day with respect to the day of inoculation. It has been done to find bacterial population. The optical densities of two strains has been observed.

\subsection{Quantitative estimation of soluble phosphate:}

Each broth culture supernatant has been centrifuged at $10000 \mathrm{rpm}$ for 10 minutes. IT has been followed by filteration through 0.22 micrometre nitrocellulose membrane. The procedure has been carried out on every other day starting from 2nd to 18th day with respect to the day of inoculation. Simultenusly, each broth culture supernatant has been used for quantitative assessment of soluble phosphate as degraded part of phorate. Then, the soluble phosphate has been measured by standard protocol of Murphy and Reily [7].

\subsection{Kinetics for biodegradation of phorate:}

Phorate biodegradation -kinetics has been studied to find the biodegradation of rate of insoluble phosphate concentration. The following equation has been used:

$\mathrm{K}=2.302 \times(\log \mathrm{Ct}-\log \mathrm{C} 0) / \mathrm{t}$, Where, $\mathrm{K}=$ Constant rate and $\mathrm{t}=$ Time. $\mathrm{C} 0$ is the phosphate concentration at $\mathrm{t}=0 . \mathrm{Ct}$ is the concentration of soluble phosphate after time $t$.

\subsection{LC-MS analysis:}

The culture supernatant of the 12th day has been used in Liquid Chromatography and mass Spectrometry ( LC-MS) for analysis of of biodegradable parts of phorate in presence of other constituent of Pikovskaya media. The combination of $0.1 \%$ formic acid with methanol ( $32: 68, \mathrm{v} / \mathrm{v}$ ) has been used as the mobile phase.

\subsection{Plant growth promotion test:}

The culture of the 16th day has been used as bio-inoculants for sterilized soil in culture tube and incubated at room temperature ( $300 \mathrm{C}$ ) for a week. The prior to the beginning of the procedure, the Bengal gram seeds ( Cier arietinum) have been surface sterilized, soaked in sterilized distilled water for 4 hours, and incubated on the treated soil. In addition, the treated seeds have been kept at room temperature ( 30) C) for 15 days. The number of germinated seeds has been counted on the 2 nd day and the root-shoot development has been observed on the 15 th day.

\section{RESULTS AND DISCUSSION}

The data obtained after doing some experiments and analysis of the samples, can be used to study the application of bacteria culture to control contaminated toxic insecticides and pesticides applied by the farmer in the agricultural lands.

\subsection{Change in $\mathrm{pH}$ in broth culture:}

From the experimental data, it has been found that $\mathrm{pH}$ of the supernatant of the two bacterial cultures have become acidic in nature with respect to the control. The acidic nature is due to the production of organic acids and phosphoric acids.

\subsection{Effect on bacterial growth:}

Three cases have been considered for assessment of bacterial growth. In first case, ammonium molybdate has been added in normal modified Pikovskya media. In second case, molybdenum salt has been added in the normal modified Pikovskya media. In the last case, no molybdenum salt has been added. From the experimental data it has been seen that the lower concentration, $0.01 \mathrm{M} / \mathrm{L}$, of molybdenum salts of ammonium and sodium has not contributed in the significant increase of bacterial growth for the strain, E57. However, it has been observed that the addition of lower concentration of ammonium molybdenum has slightly increased the growth rate of ZS20 strain. The same result has not been achieved with lower concentration of sodium molybdenum. Addition of higher concentration, $0.12 \mathrm{M} / \mathrm{L}$, of molybdenum salts to two bacterial strains has highly increased their biomass. On a scale of comparison, , it has been found that the first case performed better than the second one. The third case has not produced any significant result due to the absence of molybdenum salts. It has been also observed that after an average of 14 days, the bacterial growth rates of the two strains gradually decrease. On the 4th day, both the cultures have entered their log phases and stayed in stationary phases for an average of 14 days. At the end of stationary phases, the bacterial cultures entered their death phases.

\subsection{Phorate degradation into phosphate:}

It has been found from the experiment that only the bacterial cultures of E57 and ZS20 are sufficient enough for phorate degradation. However, the bacterial strains have been able to degrade phorate with the addition of ammonium molybdate or 
sodium molybdate. Lower concentration of the both the salts have partially degraded the phorate, whereas the higher concentration of both the salts have significantly degraded the phorate into soluble phosphate.. In an average, soluble phosphate concentration increases from 8th to 12th day. Again, the performance of phorate degradation of ZS20 has been found to be better than that of E57. Also, ammonium molybdate has been effective catalyst than that of sodium molybdate. The available phosphate in the supernatant is due to the degradation of phorate by bacterial cultures.

\subsection{Variation of kinetic constant for available phosphate:}

It has been observed that throughout the experiment, the rate constant, $\mathrm{K}$, for first order kinetics is inversely proportional to the available soluble phosphate. An increase in the soluble phosphate concentration, higher rate of phorate degradation, decreases the rate constant value and vice-versa.

\subsection{LC-MS analysis:}

The LC-MS data has indicated the presence of the degraded part of phorate, ammonium molybdate, and other components of the media. The peak of phorate has been detected in the control sample containing all constituents of modified Pikovskaya media along with the ammonium molybdate but without bacterial cultures.

\subsection{Plant growth activity test:}

It has been found from the experimental results that germination of Cier arietinum seeds is nil when seeds are taken with i) PVK media containing phorate, ii) PVK media containing phorate and ZS20 strain, iii) PVK containing media containing phorate, lower concentration of ammonium molybdate and E57 strain respectively. But greater and better performance has been found for the rest of the sample with respect to the control. Here, the rate of seedling germination percentage, vigorindex, dry weight of the plantlet has significantly increased. The higher concentration of molybdate salts of ammonium and sodium has acted as catalyst for degradation of phorate into soluble phosphate. The soluble phosphate obtained from the degradation of phorate is very useful for growth of the plants. The toxic effects of phorate have been reduced by the bacterial cultures to form eco-friendly environment for plantlet. The addition of higher concentration of molybdate salts has produced better results than lower concentration of the salts or addition of no molybdate salts. Since, ammonium molybdate produces huge amount of phosphatase enzyme and organic acids, the combined effect of these organic acids and enzyme helps for complete degradation of insoluble non-toxic phosphate compound.

\section{CONCLUSION}

The phorate can be degraded by Enterobacter and Pseudomonas sp. When the $\mathrm{pH}$ ranges of bacterial cultures are below 7. Such bacterial strains can act in acidic soil for phorate degradation and help to maiNtain its fertility after detoxification. Thus pollution of agricultural lands can be controlled. In addition, it can supply soluble phosphate as plant nutrient. Molybdate ion can act as a sensor. It can sensitize the phosphate degradation bacteria for secretion for organic acids and phosphatase enzyme. The combined effect of ammonium molybdate salt, enzyme, and organic acids helps for complete degradation of water insoluble toxic phorate which causes land pollution, into soluble non-toxic phosphate compounds which is pollution free.

\section{REFERENCES}

[1] Rana Gopinath, Mandal Tanusri, Sakha Dhruba, Mandal N. K. and Meikap B. C, "Calcite solubilization by Bacteria: A novel method of Environmental pollution control", Geomicrobiology Journal (Taylor and Francis ), vo. 14, pp. 846-852 (2015).

[2] Saha Sangeeta and Mandal Tanusri, "Characterisation of Insoluble organophosphate degrading bacteria isolated from root of citrus plant". International Journal of Current Research and Review. Vol. 9(13), pp. 38 -44 (2017).

[3] Saha Sangeeta, Rana Gopinath, . Mukhopadhaya A. and Mandal Tanusri," Study of the catalytic effect of Molybdenum salt on bacteria for phorate degradation". Journal of Biological and Physical Sciences, vol.7(2), pp. 519 -536(2017).

[4] Saha Sangeeta, Gopinath Rana, . Mukhopadhya A., Mandal A. and Mandal Tanusri, "Integrated catalytic system between the biological non-biological materials for bio-degradation of toxic methyl-paratan into non-toxic compounds". Asian Journal of of Microbiology, Biotechnology and Environmental Sciences, vol.20(1), pp. $238-245(2018)$.

[5] Saha Sangeeta, Rana Gopinatn, Mukhopadhya A. and Manal Tanusri, " Conversion of un-used organophosphate containing pesticide into bioavailable nutrient by eco-friendly bacteria”, Proc. Of International Conference on Frontier Discoveries and Emerging opportunities in Life Science (FDEOLS-2014), India.

[6] Rodriguez H, Fraga R., “ Phosphate solubilizing bacteria and their role in plant-growth promotion”. Biotechnology Advances, vol.17940, pp.319 339(1999).

[7] Murpy J, Riley J.P. 'A modified single solution method for determination of phosphate in natural water. Anal. Chim. Acta, pp. 31-36 (1962).

[8] Mohamadi K. 'Phosphorus solubilizing bacteria: Occurancre, Mechanism, and their role in crop production'. Resource and Environment. Vol. 2(1), pp. 80-85 (2012).

[9] Sharma S. B., Sayyed R. Z., Tribedi M.H. and Gobl T.A. 'Phosphate solubilizing microbes: Sustainable approach for managing phosphorus deficiency in agricultural soils'. Springer Plus (2013). 